DARIUSz ZAWisza (Kraków)

\title{
ROBUST PORTFOLIO SELECTION UNDER EXPONENTIAL PREFERENCES
}

Abstract. We consider an incomplete market with an untradable stochastic factor and a robust investment problem based on the CARA utility. We formulate it as a stochastic differential game problem, and use HamiltonJacobi-Bellman-Isaacs equations to derive an explicit representation of the robust optimal portfolio; the HJBI equation is transformed using a substitution of the Cole-Hopf type. Not only the pure investment problem, but also a problem of robust hedging is taken into account: an agent tries to hedge the risk associated with derivatives based on the stochastic factor.

1. Introduction. The purpose of this paper is to describe an optimal financial strategy which an agent can follow in order to manage his model risk. The agent trades between a riskless bond and a risky asset whose price is a diffusion with dynamics affected by a correlated untradable stochastic factor. It is worth mentioning that this model includes stochastic volatility models, and it is usually used to describe weather influence on electric and gas prices.

The classical optimality criterion is based on an expected utility functional of the form

$$
X \mapsto \mathbb{E}(U(X)) .
$$

Instead of supposing that the trader knows the exact model followed by the real market, we assume here that the trader knows that the correct model belongs to a wide class of models. Here this class is represented by a set $\mathcal{Q}$ of equivalent measures. Therefore, it is reasonable to consider an optimality

2010 Mathematics Subject Classification: 91G10, 91A15, 91A23, 49L99, 93E20.

Key words and phrases: robust control, stochastic differential games, model uncertainty, exponential utility. 
criterion based on a robust optimality functional

$$
X \mapsto \inf _{Q \in \mathcal{Q}} \mathbb{E}(U(X)) .
$$

Maximization of the robust functional was considered, among others, by Fölmer and Gundel [3, Gundel [5], Hernández and Schied [6], Korn and Menkens [7, Korn and Wilmott [8], Mataramvura and Øksendal [9], Øksendal and Sulem [12], [11], Schied [15], Schied and Wu [16], and Talay and Zheng [18. Some of these papers are based on duality arguments. In this work, however, the emphasis is on the value function and not on optimal strategies. Our paper is closest to Hernández and Schied's paper [6], where they use a specific class of risk preferences, namely the HARA utility function $\left(U(x)=x^{\gamma}\right)$. This allows them to combine duality results of Schied and $\mathrm{Wu}[15]$ with the stochastic control approach and determine a robust optimal strategy.

In our work we consider the same model, but assumptions concerning coefficients are more general, and instead of HARA we use the CARA (Constant Absolute Risk Aversion) utility $\left(U(x)=-e^{-\gamma x}\right)$. In addition, we do not limit ourselves to finding a pure robust optimal strategy. We consider claims based on the stochastic factor and try to find a robust strategy which is able to hedge away the risk associated with this claim. Such derivatives involving catastrophic damages, weather and volatility derivatives are attractive in many economic activities.

Our solution of the robust investment problem is based on stochastic differential game theory and Hamilton-Jacobi-Bellman-Isaacs equations. The associated HJBI equation can be simplified by applying transformations of the Cole-Hopf type. The resulting equation can be regarded as a classical HJB equation. Therefore, its solutions have a stochastic representation. As a by-product we obtain a formula for the optimal strategy. In our opinion the method presented in our paper works faster than the duality theory and can be applied not only to the CARA utility but also to other utilities. Besides, one might have difficulty applying duality results to the exponential utility, because it fails to satisfy the Inada condition $\left(U^{\prime}(0)=+\infty\right.$, $\left.U^{\prime}(+\infty)=0\right)$.

Let us recall that the methodology based on the Cole-Hopf transformations was developed by Zariphopoulou [20], 21], Musiela and Zariphopoulou [10], and Pham [13. We should also mention that HJBI equations were successfully applied to robust optimal investment problems by Mataramvura and Øksendal [9], Øksendal and Sulem [12], 11], and Talay and Zhang [18], but to our knowledge this is the first time they are used in models with an untradable risk factor to obtain an explicit formula for a robust optimal strategy. 
2. Model and main result. Let $(\Omega, \mathcal{F}, P)$ be a probability space with a filtration $\left(\mathcal{F}_{t}, 0 \leq t \leq T\right)$ generated by two independent Brownian motions $\left(W_{t}^{1}, 0 \leq t \leq T\right),\left(W_{t}^{2}, 0 \leq t \leq T\right)$. We consider an incomplete financial market consisting of two tradable assets $\left(B_{t}, 0 \leq t \leq T\right)$ and $\left(S_{t}, 0 \leq t \leq T\right)$ and one untradable factor $\left(Y_{t}, 0 \leq t \leq T\right)$. $B_{t}$ is a risk-free asset, and $S_{t}$ is a risky asset. These processes are solutions of the system of stochastic differential equations

$$
\left\{\begin{array}{l}
d B_{t}=r B_{t} d t \\
d S_{t}=b\left(Y_{t}\right) S_{t} d t+\sigma\left(Y_{t}\right) S_{t} d W_{t}^{1}, \\
d Y_{t}=g\left(Y_{t}\right) d t+\rho d W_{t}^{1}+\sqrt{1-\rho^{2}} d W_{t}^{2} .
\end{array}\right.
$$

The coefficients $b, \sigma>0, g$ are continuous functions and they are assumed to satisfy all the required regularity conditions, in order to guarantee the existence of a unique strong solution to 2.1. $r>0$ is the interest rate and $\rho \in(-1,1)$ is the correlation coefficient. Let $\lambda$ denote the risk premium ratio

$$
\lambda(y)=\frac{b(y)}{\sigma(y)} .
$$

The time-independence of the coefficients is for notational convenience only and can easily be relaxed.

REMARK. Pham 13] noticed that the identity condition on the diffusion term of $Y$ is not very restrictive. Suppose we have a dynamics for $Y$ in the form

$$
d Y_{t}=g\left(Y_{t}\right) d t+a\left(Y_{t}\right)\left(\rho d W_{t}^{1}+\sqrt{1-\rho^{2}} d W_{t}^{2}\right) .
$$

Then, by assuming that there exists $f \in \mathcal{C}^{2}(\mathbb{R})$ such that $f^{\prime}(y)=1 / a(y)$ we see, by the Itô formula, that the diffusion term of $f\left(Y_{t}\right)$ is identity. However, after that change of variable the required regularity conditions are not always satisfied.

The agent's risk preferences are captured by a utility function. We use the utility function of the CARA (Constant Absolute Risk Aversion) type

$$
U(x)=-e^{-\gamma x},
$$

where $\gamma>0$ is the risk aversion parameter.

The dynamics of the agent's wealth process $\left(\bar{X}_{t}^{\bar{\pi}}, 0 \leq t \leq T\right)$ is given by the stochastic differential equation

$$
\left\{\begin{array}{l}
\bar{X}_{t}=\left(r \bar{X}_{t}+\bar{\pi}_{t}\left(b\left(Y_{t}\right)-r\right)\right) d t+\bar{\pi}_{t} \sigma\left(Y_{t}\right) d W_{t}^{1}, \\
\bar{X}_{s}=\bar{x},
\end{array}\right.
$$

where $\bar{x}$ denotes the current wealth of the agent. It is more convenient to rewrite the above equation in terms of forward values. More precisely, we denote by $X_{t}$ the $T$-forward value of the wealth, i.e.

$$
X_{t}=e^{(T-t) r} \bar{X}_{t}, \quad \pi_{t}=e^{(T-t) r} \bar{\pi}_{t} .
$$


Then the dynamics of the wealth process can be rewritten as

$$
d X_{t}=\pi_{t}\left(b\left(Y_{t}\right)-r\right) d t+\pi_{t} \sigma\left(Y_{t}\right) d W_{t}^{1} .
$$

Without loss of generality we can assume that the interest rate $r$ is equal to 0 , which gives us

$$
d X_{t}=\pi_{t} b\left(Y_{t}\right) d t+\pi_{t} \sigma\left(Y_{t}\right) d W_{t}^{1} .
$$

Definition 2.1. A control (or strategy) $\pi=\left(\pi_{t}, s \leq t \leq T\right)$ is admissible on the time interval $[s, T]$, written $\pi \in \mathcal{A}_{s}$, if it satisfies the following assumptions:

(1) $\pi$ is progressively measurable with respect to the filtration $\left(\mathcal{F}_{t}, s \leq\right.$ $t \leq T)$

(2) the stochastic process 2.2 exists and

$$
\mathbb{E}_{x, y, s} \sup _{s \leq t \leq T} \exp \left(-2 \gamma X_{t}^{\pi}\right)<+\infty
$$

for each $(x, y) \in \mathbb{R}^{2}$.

The admissible control $\pi$ can be interpreted as the part of the agent's wealth invested in $S_{t}$. Note that $\pi$ is allowed to be negative.

We assume that the model is not precisely known and the agent knows only a class of possible models. Following Hernández and Schied [6], we will consider the class

$$
\mathcal{Q}:=\left\{Q \sim P \mid \frac{d Q}{d P}=\mathcal{E}\left(\int \eta_{t 1} d W_{t}^{1}+\eta_{t 2} d W_{t}^{2}\right)_{T},\left(\eta_{1}, \eta_{2}\right) \in \mathcal{M}\right\},
$$

where $\mathcal{E}(\cdot)_{t}$ denotes the Doléans-Dade exponential and $\mathcal{M}$ denotes the set of all progressively measurable processes $\eta=\left(\eta_{1}, \eta_{2}\right)$ taking values in a fixed compact convex set $\Gamma \subset \mathbb{R}^{2}$. The measure determined by $\eta \in \mathcal{M}$ is denoted by $Q^{\eta}$.

Statement of the problem. Let $\beta$ be a continuous function. $\beta\left(Y_{T}\right)$ is a derivative payoff based on the factor $\left(Y_{t}, 0 \leq t \leq T\right)$. The agent tries to build a robust portfolio to hedge the risk associated with this claim, which means that the objective of the agent (seller of the claim) is to

$$
\operatorname{maximize} \inf _{Q \in \mathcal{Q}} J_{\beta}^{\pi, Q}(x, y, t) \quad \text { over } \pi \in \mathcal{A}_{t},
$$

where

$$
J_{\beta}^{\pi, Q}(x, y, t)=\mathbb{E}_{x, y, t}^{Q}\left(U\left(X_{T}^{\pi}-\beta\left(Y_{T}\right)\right)\right) .
$$

We can consider this as a zero-sum stochastic differential game problem. The measure $Q$ is the control of player 1 (the "market"), while the portfolio $\pi$ is the control of player 2 (the "agent"). Our aim is to find the saddle point and the value function of that game. More precisely, we are looking for 
$\left(\pi^{*}, Q^{*}\right) \in \mathcal{A}_{t} \times \mathcal{Q}$ and $V(x, y, t)$ such that

$$
J_{\beta}^{\pi, Q^{*}}(x, y, t) \leq J_{\beta}^{\pi^{*}, Q^{*}}(x, y, t) \leq J_{\beta}^{\pi^{*}, Q}(x, y, t),
$$

and

$$
V(x, y, t)=\sup _{\pi \in \mathcal{A}_{t}} \inf _{Q \in \mathcal{Q}} J_{\beta}^{\pi, Q}(x, y, t)=\inf _{Q \in \mathcal{Q}} \sup _{\pi \in \mathcal{A}_{t}} J_{\beta}^{\pi, Q}(x, y, t)=J_{\beta}^{\pi^{*}, Q^{*}}(x, y, t) .
$$

Definition 2.2. A pair of controls $\left(\pi^{*}, Q^{*}\right) \in \mathcal{A}_{t} \times \mathcal{Q}$ is called an optimal control if it satisfies 2.3 for all $\pi \in \mathcal{A}_{t}$ and $Q \in \mathcal{Q}$.

From classical stochastic control theory we know that an optimal control is usually given in the feedback form $\left(\left(\pi\left(X_{t}, Y_{t}, t\right), \eta\left(X_{t}, Y_{t}, t\right)\right), 0 \leq t \leq T\right)(\eta$ determines the measure $\left.Q^{\eta}\right)$. Such controls are often called Markov controls and are denoted simply by $(\pi(x, y, t), \eta(x, y, t))$. For more information about differential games we refer to Fleming and Soner [2] and references therein.

We summarize our main result in the following theorem:

THEOREM 2.3. Let $g, \beta, \lambda, \lambda^{2}$ be bounded and uniformly Lipschitz continuous functions. Then

$$
\sup _{\pi \in \mathcal{A}_{t}} \inf _{Q \in \mathcal{Q}} J_{\beta}^{\pi, Q}(x, y, t)=\inf _{Q \in \mathcal{Q}} \sup _{\pi \in \mathcal{A}_{t}} J_{\beta}^{\pi, Q}(x, y, t)=-e^{-\gamma x}(\alpha(y, t))^{1 /\left(1-\rho^{2}\right)},
$$

where $\alpha$ is the solution of the equation

$$
\begin{aligned}
\alpha_{t} & +\frac{1}{2} \alpha_{y y}+(g(y)-\rho \lambda(y)) \alpha_{y} \\
& +\max _{\eta_{1}, \eta_{2} \in \Gamma}\left(-\frac{1-\rho^{2}}{2}\left(\lambda(y)+\eta_{1}\right)^{2} \alpha+\sqrt{1-\rho^{2}} \eta_{2} \alpha_{y}\right)=0
\end{aligned}
$$

with the terminal condition

$$
\alpha(y, T)=\exp \left(\left(1-\rho^{2}\right) \gamma \beta(y)\right) .
$$

Moreover, there exists an optimal pair of controls $\left(\eta_{1}^{*}(y, t), \eta_{2}^{*}(y, t)\right)$ such that $\eta^{*}$ realizes the maximum in (2.4), and the agent's strategy is given by

$$
\pi^{*}(x, y, t)=\frac{\rho}{\gamma \sigma(y)} \frac{F_{y}}{F}+\frac{\lambda(y)+\eta_{1}^{*}(y, t)}{\gamma \sigma(y)},
$$

where

$$
F(y, t)=(\alpha(y, t))^{1 /\left(1-\rho^{2}\right)} .
$$

3. HJBI equations and the verification theorem. The robust investment problem stated in the previous section can be solved by applying stochastic control theory and the Hamilton-Jacobi-Bellman-Isaacs equation. Let $\mathcal{L}$ denote the differential operator given by

$$
\begin{aligned}
\mathcal{L}^{\pi \eta} V(x, y, t)= & V_{t}+\frac{1}{2} V_{y y}+\frac{1}{2} \pi^{2} \sigma^{2}(y) V_{x x}+\rho \pi \sigma(y) V_{x y} \\
& +\pi\left(b(y)+\eta_{1} \sigma(y)\right) V_{x}+\left(\rho \eta_{1}+\sqrt{1-\rho^{2}} \eta_{2}\right) V_{y}+g(y) V_{y} .
\end{aligned}
$$


Next we modify the verification theorem proposed in Mataramwura and Øksendal [9] to make it applicable to our problem.

TheOrem 3.1. Suppose there exist a function $V \in \mathcal{C}^{2,2,1}\left(\mathbb{R}^{2} \times[0, T)\right) \cap$ $\mathcal{C}\left(\mathbb{R}^{2} \times[0, T]\right)$ and an admissible Markov control $\left(\pi^{*}(x, y, t), \eta^{*}(x, y, t)\right)$ such that

$$
\begin{aligned}
& \mathcal{L}^{\pi^{*}(x, y, t), \eta} V(x, y, t) \geq 0, \\
& \mathcal{L}^{\pi, \eta^{*}(x, y, t)} V(x, y, t) \leq 0, \\
& \mathcal{L}^{\pi^{*}(x, y, t), \eta^{*}(x, y, t)} V(x, y, t)=0, \\
& V(x, y, T)=U(x-\beta(y))
\end{aligned}
$$

for all $\eta \in \Gamma, \pi \in \mathbb{R},(x, y, t) \in \mathbb{R}^{2} \times[0, T)$, and

$$
\mathbb{E}_{x, y, t}^{Q}\left(\sup _{t \leq s \leq T}\left|V\left(X_{s}^{\pi}, Y_{s}, s\right)\right|\right)<+\infty
$$

for all $(x, y, t) \in \mathbb{R}^{2} \times[0, T], \pi \in \mathcal{A}_{t}$, and $Q \in \mathcal{Q}$. Then

$$
J_{\beta}^{\pi, Q^{*}}(x, y, t) \leq V(x, y, t) \leq J_{\beta}^{\pi^{*}, Q}(x, y, t)
$$

for all $\pi \in \mathcal{A}_{t}$ and $Q \in \mathcal{Q}$, and

$$
V(x, y, t)=J_{\beta}^{\pi^{*}, Q^{*}}(x, y, t) .
$$

REMARK. Let us point out that conditions (3.1) - 3.4 hold if the following Hamilton-Jacobi-Bellman-Isaacs equations are satisfied:

$$
\begin{aligned}
\max _{\pi \in \mathbb{R}} \min _{\eta \in \Gamma} \mathcal{L}^{\pi, \eta} V(x, y, t) & =\min _{\eta \in \Gamma} \max _{\pi \in \mathbb{R}} \mathcal{L}^{\pi, \eta} V(x, y, t)=0, \\
V(x, y, T) & =U(x-\beta(y)) .
\end{aligned}
$$

Proof of Theorem 3.1. Choose any $\eta \in \mathcal{M}$ and consider the system

$$
\left\{\begin{array}{l}
d X_{t}=\pi^{*}\left(X_{t}, Y_{t}, t\right) b\left(Y_{t}\right) d t+\pi^{*}\left(X_{t}, Y_{t}, t\right) \sigma\left(Y_{t}\right) d W_{t}^{1}, \\
d Y_{t}=g\left(Y_{t}\right) d t+\rho d W_{t}^{1}+\sqrt{1-\rho^{2}} d W_{t}^{2} .
\end{array}\right.
$$

It is convenient to consider the $Q^{\eta}$-dynamics of the system (3.8). After applying the Girsanov transformation, we have

$$
\left\{\begin{array}{l}
d X_{t}=\pi_{t}^{*}\left(b\left(Y_{t}\right)+\eta_{t} \sigma\left(Y_{t}\right)\right) d t+\pi_{t}^{*} \sigma\left(Y_{t}\right) d W_{t}^{\eta 1}, \\
d Y_{t}=\left(g\left(Y_{t}\right)+\eta_{t 1} \rho+\eta_{t 2} \sqrt{1-\rho^{2}}\right) d t+\rho d W_{t}^{\eta 1}+\sqrt{1-\rho^{2}} d W_{t}^{\eta 2},
\end{array}\right.
$$

where $\pi_{t}^{*}=\pi^{*}\left(X_{t}, Y_{t}, t\right)$ and $\left(W_{s}^{\eta 1}, W_{s}^{\eta 2}\right)$ are $Q^{\eta}$-Brownian motions given by

$$
\left\{\begin{array}{l}
d W_{t}^{\eta 1}=d W_{t}^{1}-\eta_{t 1} d t \\
d W_{t}^{\eta 2}=d W_{t}^{2}-\eta_{t 2} d t
\end{array}\right.
$$


If we apply the Itô formula to $(3.9)$ and the function $V$, we get

$$
\begin{aligned}
\mathbb{E}_{x, y, t}^{Q^{\eta}}\left(V \left(X_{T_{n}}^{\pi^{*}}, Y_{T_{n}},\right.\right. & \left.\left.T_{n}\right)\right)=V(x, y, t)+\mathbb{E}_{x, y, t}^{Q^{\eta}} \int_{t}^{T_{n}} \mathcal{L}^{\pi_{s}^{*}, \eta_{s}} V\left(X_{s}^{\pi^{*}}, Y_{s}, s\right) d s \\
& +\mathbb{E}_{x, y, t}^{Q^{\eta}}\left(\int_{t}^{T_{n}}\left(\pi_{s}^{*} \sigma\left(Y_{s}\right) V_{x}\left(X_{s}^{\pi^{*}}, Y_{s}, s\right)+\rho V_{y}\left(X_{s}^{\pi^{*}}, Y_{s}, s\right)\right) d W_{s}^{1}\right. \\
& \left.+\int_{t}^{T_{n}} \sqrt{1-\rho^{2}} V_{y}\left(X_{s}^{\pi^{*}}, Y_{s}, s\right) d W_{s}^{2}\right),
\end{aligned}
$$

where $\left(T_{n}, n=1,2, \ldots\right)\left(T_{n} \rightarrow T, T_{n}<T\right)$ is a localizing sequence of stopping times such that

$$
\begin{aligned}
\mathbb{E}_{x, y, t}^{Q^{\eta}}\left(\int _ { t } ^ { T _ { n } } \left(\pi_{s}^{*} \sigma\left(Y_{s}\right) V_{x}\left(X_{s}^{\pi^{*}}, Y_{s}, s\right)\right.\right. & \left.+\rho V_{y}\left(X_{s}^{\pi^{*}}, Y_{s}, s\right)\right) d W_{s}^{1} \\
& \left.+\int_{t}^{T_{n}}\left(\sqrt{1-\rho^{2}} V_{y}\left(X_{s}^{\pi^{*}}, Y_{s}, s\right)\right) d W_{s}^{2}\right)=0 .
\end{aligned}
$$

Using (3.1) yields

$$
\mathbb{E}_{x, y, t}^{Q^{\eta}}\left(V\left(X_{T_{n}}^{\pi^{*}}, Y_{T_{n}}, T_{n}\right)\right) \geq V(x, y, t) .
$$

Since (3.5) holds, we can apply the dominated convergence theorem. Letting $n \rightarrow+\infty$ and using (3.4) we obtain

$$
V(x, y, t) \leq J_{\beta}^{\pi^{*}, Q}(x, y, t) .
$$

If we replace $\eta$ by $\eta^{*}$ and apply (3.3), we have

$$
V(x, y, t)=J_{\beta}^{\pi^{*}, Q^{*}}(x, y, t) .
$$

Next we choose $\pi \in \mathcal{A}_{t}$ and apply the Itô formula to the system

$$
\left\{\begin{array}{l}
d X_{t}=\pi_{t}\left(b\left(Y_{t}\right)+\eta_{t}^{*} \sigma\left(Y_{t}\right)\right) d t+\pi_{t} \sigma\left(Y_{t}\right) d W_{t}^{\eta^{*} 1}, \\
d Y_{t}=\left(g\left(Y_{t}\right)+\eta_{t 1}^{*} \rho+\eta_{t 2}^{*} \sqrt{1-\rho^{2}}\right) d t+\rho d W_{t}^{\eta^{*} 1}+\sqrt{1-\rho^{2}} d W_{t}^{\eta^{*} 2} .
\end{array}\right.
$$

Repeating the method presented above and using (3.2) we get

$$
V(x, y, t) \geq J_{\beta}^{\pi, Q^{*}}(x, y, t) .
$$

4. Derivation of the optimal strategy. To verify the assumptions of Theorem 3.1 we proceed to construct a candidate solution of the HJBI equations (3.6) and proper controls $\left(\pi^{*}, \eta^{*}\right)$. Let us consider first the equation

$$
\min _{\eta \in \Gamma} \max _{\pi \in \mathbb{R}} \mathcal{L}^{\pi, \eta} V(x, y, t)=0,
$$


i.e.

$$
\begin{aligned}
V_{t}+ & \frac{1}{2} V_{y y}+\min _{\left(\eta_{1}, \eta_{2}\right) \in \Gamma} \max _{\pi \in \mathbb{R}}\left(\frac{1}{2} \pi^{2} \sigma^{2}(y) V_{x x}+\rho \pi \sigma(y) V_{x y}\right. \\
& \left.+\pi\left(b(y)+\eta_{1} \sigma\right) V_{x}+\left(\rho \eta_{1}+\sqrt{1-\rho^{2}} \eta_{2}\right) V_{y}\right)+g(y) V_{y}=0 .
\end{aligned}
$$

The utility function of the exponential type suggests that we should be interested in the following terminal condition:

$$
V(x, y, T)=\exp (-\gamma x+\gamma \beta(y)) .
$$

In order to demonstrate the key calculations we assume that all the required derivatives of the relevant solutions exist. The rigorous result together with necessary assumptions on the market coefficients is given later. Calculations of the type presented below are commonly used in the existing literature (see Musiela and Zariphopoulou [10] or Benth and Karlsen [1]) and are not given here with all details.

Notice that if $V_{x x}<0$ then the maximum over $\pi$ in 4.1 is well defined. It is achieved at

$$
\pi^{*}(x, y, t, \eta)=-\frac{\rho}{\sigma(y)} \frac{V_{x y}}{V_{x x}}-\frac{b(y)+\eta_{1} \sigma(y)}{\sigma^{2}(y)} \frac{V_{x}}{V_{x x}} .
$$

Following Musiela and Zariphopoulou [10] we observe that the terminal condition 4.2 suggest that the solution should be of the form

$$
V(x, y, t)=-e^{-\gamma x} F(y, t)
$$

Therefore,

$$
\pi^{*}(x, y, t, \eta)=\frac{\rho}{\gamma \sigma(y)} \frac{F_{y}}{F}+\frac{\lambda(y)+\eta_{1}}{\gamma \sigma(y)} .
$$

Substituting 4.5 and 4.4 in 4.1 shows that $F$ solves

$$
\begin{aligned}
F_{t}-\frac{1}{2} \rho^{2} \frac{F_{y}^{2}}{F} & +\frac{1}{2} F_{y y}+(g(y)-\rho \lambda(y)) F_{y} \\
& +\max _{\left(\eta_{1}, \eta_{2}\right) \in \Gamma}\left(-\frac{1}{2}\left(\lambda(y)+\eta_{1}\right)^{2} F+\sqrt{1-\rho^{2}} \eta_{2} F_{y}\right)=0
\end{aligned}
$$

together with the terminal condition

$$
F(y, T)=\exp (\gamma \beta(y))
$$

To remove the nonlinear term $F_{y}^{2} / F$ we make the following Cole-Hopf type transformation:

$$
F(y, t)=(\alpha(y, t))^{1 /\left(1-\rho^{2}\right)} .
$$


Differentiating yields

$$
\begin{aligned}
F_{t} & =\frac{1}{1-\rho^{2}} \alpha_{t} \cdot \alpha^{\rho^{2} /\left(1-\rho^{2}\right)}, \\
F_{y} & =\frac{1}{1-\rho^{2}} \alpha_{y} \cdot \alpha^{\rho^{2} /\left(1-\rho^{2}\right)}, \\
F_{y y} & =\frac{1}{1-\rho^{2}} \frac{\rho^{2}}{1-\rho^{2}} \alpha_{y}^{2} \cdot \alpha^{1 /\left(1-\rho^{2}\right)-2}+\alpha_{y y} \cdot \alpha^{\rho^{2} /\left(1-\rho^{2}\right)} .
\end{aligned}
$$

Substituting the above derivatives in 4.6 gives

$$
\begin{aligned}
\alpha_{t}+\frac{1}{2} \alpha_{y y}+(g(y)-\rho \lambda(y)) \alpha_{y} \\
\quad+\max _{\left(\eta_{1}, \eta_{2}\right) \in \Gamma}\left(-\frac{1-\rho^{2}}{2}\left(\lambda(y)+\eta_{1}\right)^{2} \alpha+\sqrt{1-\rho^{2}} \eta_{2} \alpha_{y}\right)=0
\end{aligned}
$$

and

$$
\alpha(y, T)=\exp \left(\left(1-\rho^{2}\right) \gamma \beta(y)\right) .
$$

REMARK. Note that the above equation is a quasi-linear parabolic differential equation of the second order and has the form of a HJB equation. This fact is used to derive a stochastic representation of its solution.

Now we are ready to prove a version of the minimax theorem needed to ensure that the $V$ determined above is a solution not only to the equation

$$
\min _{\eta \in \Gamma} \max _{\pi \in \mathbb{R}} \mathcal{L}^{\pi, \eta} V(x, y, t)=0
$$

but also to

$$
\max _{\pi \in \mathbb{R}} \min _{\eta \in \Gamma} \mathcal{L}^{\pi, \eta} V(x, y, t)=0 .
$$

Proposition 4.1. Suppose that there exist a positive solution $\alpha$ of the problem 4.8 -4.9 and let the maximum in (4.8) be attained at $\eta^{*}(y, t)=$ $\left(\eta_{1}^{*}(y, t), \eta_{2}^{*}(y, t)\right)$. Then

$$
\max _{\pi \in \mathbb{R}} \min _{\eta \in \Gamma} \mathcal{L}^{\pi, \eta} V(x, y, t)=\min _{\eta \in \Gamma} \max _{\pi \in \mathbb{R}} \mathcal{L}^{\pi, \eta} V(x, y, t)=\mathcal{L}^{\pi^{*}, \eta^{*}} V(x, y, t)=0,
$$

where

$$
V(x, y, t)=-e^{-\gamma x} F(y, t)=-e^{-\gamma x}(\alpha(y, t))^{1 /\left(1-\rho^{2}\right)},
$$

and

$$
\pi^{*}(x, y, t)=\frac{\rho}{\gamma \sigma(y)} \frac{F_{y}}{F}+\frac{\lambda(y)+\eta_{1}^{*}(y, t)}{\gamma \sigma(y)} .
$$

Proof. Since we always have

$$
\max _{\pi \in \mathbb{R}} \min _{\eta \in \Gamma} \mathcal{L}^{\pi, \eta} V(x, y, t) \leq \min _{\eta \in \Gamma} \max _{\pi \in \mathbb{R}} \mathcal{L}^{\pi, \eta} V(x, y, t),
$$


it is sufficient to prove that

$$
\min _{\eta \in \Gamma} \mathcal{L}^{\pi^{*}, \eta} V(x, y, t)=\min _{\eta \in \Gamma} \max _{\pi \in \mathbb{R}} \mathcal{L}^{\pi, \eta} V(x, y, t) .
$$

It follows from calculations (4.4)- 4.9) that the right side above is equal to

$$
\begin{aligned}
-e^{-\gamma x}\left(F_{t}+\frac{1}{2} F_{y y}-\right. & \frac{1}{2} \rho^{2} \frac{F_{y}^{2}}{F}+(g(y)-\rho \lambda(y)) F_{y} \\
& \left.+\max _{\eta \in \Gamma}\left(-\frac{1}{2}\left(\lambda(y)+\eta_{1}\right)^{2} F+\sqrt{1-\rho^{2}} \eta_{2} F_{y}\right)\right),
\end{aligned}
$$

where the maximum is achieved at $\eta^{*}=\left(\eta_{1}^{*}, \eta_{2}^{*}\right)$, while the left side is equal to

$$
\begin{aligned}
-e^{-\gamma x}\left(F_{t}+\frac{1}{2} F_{y y}-\frac{1}{2} \rho^{2} \frac{F_{y}^{2}}{F}+(g(y)-\rho \lambda(y)) F_{y}\right. \\
+\max _{\eta \in \Gamma}\left(\frac{1}{2}\left(\lambda(y)+\eta_{1}^{*}(y, t)\right)^{2} F\right. \\
\left.\left.\quad-\left(\lambda(y)+\eta_{1}^{*}(y, t)\right)\left(\lambda(y)+\eta_{1}\right) F+\sqrt{1-\rho^{2}} \eta_{2} F_{y}\right)\right) .
\end{aligned}
$$

Therefore, we need to show that

$$
\begin{aligned}
\max _{\left(\eta_{1}, \eta_{2}\right) \in \Gamma}\left(-\frac{1}{2}\left(\lambda(y)+\eta_{1}\right)^{2} F+\sqrt{1-\rho^{2}} \eta_{2} F_{y}\right) & \\
=\max _{\left(\eta_{1}, \eta_{2}\right) \in \Gamma}( & \frac{1}{2}\left(\lambda(y)+\eta_{1}^{*}(y, t)\right)^{2} F \\
& \left.\quad\left(\lambda(y)+\eta_{1}^{*}(y, t)\right)\left(\lambda(y)+\eta_{1}\right) F+\sqrt{1-\rho^{2}} \eta_{2} F_{y}\right) .
\end{aligned}
$$

Suppose that $F_{y} \neq 0$ and the first maximum is attained on the parabola

$$
-\frac{1}{2}\left(\lambda(y)+\eta_{1}\right)^{2} F+\sqrt{1-\rho^{2}} \eta_{2} F_{y}=C^{*}(y, t) .
$$

Then the line

$$
\begin{array}{r}
\frac{1}{2}\left(\lambda(y)+\eta_{1}^{*}(y, t)\right)^{2} F-\left(\lambda(y)+\eta_{1}^{*}(y, t)\right)\left(\lambda(y)+\eta_{1}\right) F+\sqrt{1-\rho^{2}} \eta_{2} F_{y} \\
=C^{*}(y, t)
\end{array}
$$

is tangent to the parabola at the point $\eta^{*}$. Convexity of the set $\Gamma$ implies that the second maximum must be achieved at $\eta^{*}$ and both maxima are equal. The case $F_{y}=0$ reduces our problem to maximizing, respectively, a quadratic and a linear function on an interval. Hence, the desired equality can be easily proved. 
5. Solution of the problem and related results. Let us begin with the following

Lemma 5.1. Suppose that $g, \lambda$ are bounded and uniformly Lipschitz continuous functions, and $\beta$ is a bounded function. If there exists a bounded solution of the Cauchy problem (4.8)-4.9), then it is positive and bounded away from zero.

Proof. Let $\left(Y_{s}, t \leq s \leq T\right)$ be the unique solution of the problem

$$
\left\{\begin{array}{l}
d Y_{s}=\left(g\left(Y_{s}\right)-\rho \lambda\left(Y_{s}\right)\right) d t+\rho d W_{s}^{1}+\sqrt{1-\rho^{2}} d W_{s}^{2}, \\
Y_{t}=x
\end{array}\right.
$$

Choose any $\eta \in \mathcal{M}$ and consider the measure $Q$ given by

$$
\frac{d Q}{d P}=\mathcal{E}\left(\int \eta_{s 2} d W_{s}^{2}\right)
$$

The dynamics of $Y$ under $Q$ is given by

$$
d Y_{t}=\left(g\left(Y_{t}\right)-\rho \lambda\left(Y_{t}\right)+\sqrt{1-\rho^{2}} \eta_{2 t}\right) d t+\rho d W_{t}^{1}+\sqrt{1-\rho^{2}} d W_{t}^{2 \eta} .
$$

If we apply the Itô formula and use the standard stochastic control argument to the function $\alpha$ and the process $Y$, we get

$$
\alpha(y, t) \geq \mathbb{E}_{y, t}^{Q}\left(\exp \left(\gamma\left(1-\rho^{2}\right) \beta\left(Y_{T}\right)-\frac{1-\rho^{2}}{2} \int_{t}^{T}\left(\lambda\left(Y_{S}\right)+\eta_{2 s}\right)^{2} d s\right)\right) .
$$

Since $\beta, \lambda, \eta$ are bounded, there exists $\varepsilon>0$ such that $\alpha(y, t)>\varepsilon$ for all $(y, t) \in \mathbb{R}^{2} \times[0, T]$

To complete the preparations for the proof of our main result, we need only find a set of assumptions to ensure existence of a smooth and bounded solution to 4.8. Let us recall the classical result proved by Friedman [4].

TheOREM 5.2. Suppose that $H(t, y, u, p)$ is uniformly Lipschitz continuous in $(y, u, p)$ and uniformly Hölder continuous in $t$ in compact subsets of $[0, T] \times \mathbb{R} \times \mathbb{R} \times \mathbb{R}$ and let $H$ satisfy

$$
\begin{aligned}
& H(t, y, 0,0) \leq K \\
& H(t, y, u, 0)-H(t, y, \bar{u}, 0) \leq K(u-\bar{u}) \quad \text { if } u>\bar{u}, \\
& H(t, y, u, p)-H(t, y, \bar{u}, p) \leq K_{R}(u-\bar{u}) \quad \text { if }|u|,|\bar{u}| \leq R, u>\bar{u}, \\
& |H(t, y, u, p)-H(t, \bar{y}, u, p)| \leq K_{R}(1+|p|)|y-\bar{y}| \quad \text { if }|u| \leq R, \\
& |H(t, y, u, p)-H(t, y, u, \bar{p})| \leq K_{R}(1+|y|)|p-\bar{p}| \quad \text { if }|u| \leq R
\end{aligned}
$$

for any $R>0$ and $0 \leq t \leq T, x, \bar{x}, p, \bar{p} \in \mathbb{R}$. Moreover, let $\varphi$ be a bounded and uniformly Lipschitz continuous function. Then there exists a unique solution 
$u$ of the Cauchy problem

$$
\left\{\begin{array}{l}
u_{t}+\frac{1}{2} u_{y y}+H\left(t, y, u, u_{y}\right)=0 \\
u(y, T)=\varphi(y)
\end{array}\right.
$$

which is bounded together with the derivative $u_{y}$.

Below we summarize the results obtained so far and prove our main theorem. We rewrite it here once more.

THEOREM 5.3. Let $g, \beta, \lambda, \lambda^{2}$ be bounded and uniformly Lipschitz continuous functions. Then

$$
\sup _{\pi \in \mathcal{A}_{t}} \inf _{Q \in \mathcal{Q}} J_{\beta}^{\pi, Q}(x, y, t)=\inf _{Q \in \mathcal{Q}} \sup _{\pi \in \mathcal{A}_{t}} J_{\beta}^{\pi, Q}(x, y, t)=-e^{-\gamma x}(\alpha(y, t))^{1 /\left(1-\rho^{2}\right)},
$$

where $\alpha$ is the solution of the equation

$$
\begin{aligned}
\alpha_{t} & +\frac{1}{2} \alpha_{y y}+(g(y)-\rho \lambda(y)) \alpha_{y} \\
& +\max _{\eta_{1}, \eta_{2} \in \Gamma}\left(-\frac{1-\rho^{2}}{2}\left(\lambda(y)+\eta_{1}\right)^{2} \alpha+\sqrt{1-\rho^{2}} \eta_{2} \alpha_{y}\right)=0
\end{aligned}
$$

with terminal condition

$$
\alpha(y, T)=\exp \left(\left(1-\rho^{2}\right) \gamma \beta(y)\right) .
$$

Moreover, there exists an optimal pair of controls $\left(\eta_{1}^{*}(y, t), \eta_{2}^{*}(y, t)\right)$ such that $\eta^{*}$ realizes the maximum in (5.6), and the agent's strategy is given by

$$
\pi^{*}(x, y, t)=\frac{\rho}{\gamma \sigma(y)} \frac{F_{y}}{F}+\frac{\lambda(y)+\eta_{1}^{*}(y, t)}{\gamma \sigma(y)} .
$$

Proof. First, we need to show that there exists a solution of (5.6)- 5.7). Let us consider a function $H(t, y, \alpha, p)$ given by

$$
H\left(t, y, \alpha, \alpha_{y}\right)=\max _{\eta \in \Gamma} f\left(t, y, \alpha, \alpha_{y}, \eta\right)
$$

where

$$
\begin{aligned}
f\left(t, y, \alpha, \alpha_{y}, \eta\right)= & -\frac{1-\rho^{2}}{2}\left(\lambda(y)+\eta_{1}\right)^{2} \alpha+\sqrt{1-\rho^{2}} \eta_{2} \alpha_{y} \\
& +(g(y)-\rho \lambda(y)) \alpha_{y} .
\end{aligned}
$$

The function $f$ is linear with respect to $\alpha$ and $\alpha_{y}$. Therefore, from the assumed regularity of $\lambda$ and $g$ and the well known inequality

$$
\left|\max _{\eta} f(z, \eta)-\max _{\eta} f(\bar{z}, \eta)\right| \leq \max _{\eta}|f(z, \eta)-f(\bar{z}, \eta)|,
$$

it follows that $H$ satisfies all conditions of Theorem 5.2. Hence, there exists a unique solution $\alpha$ of the problem (5.6)-(5.7), which is bounded together with the derivative $\alpha_{y}$. In addition, Lemma 5.1 shows that this solution is positive and bounded away from zero. By the classical measurable selection 
theorem (Wagner [19, Theorem 4.1]) there exists a Borel measurable $\eta^{*} \in \Gamma$ which attains the maximum in (5.6). If we set

$$
\pi^{*}(y, t)=\frac{\rho}{\gamma \sigma(y)} \frac{F_{y}}{F}+\frac{\lambda(y)+\eta_{1}^{*}(y, t)}{\gamma \sigma(y)},
$$

then $\pi^{*} b$ and $\pi^{*} \sigma$ are bounded functions. Hence, the wealth process $\left(X_{t}^{\pi^{*}}, s \leq\right.$ $t \leq T)$ exists for all $s \in[0, T]$.

In order to prove that $\pi^{*}$ is admissible, we must show that

$$
\mathbb{E}_{x, y, t} \sup _{t \leq r \leq T} \exp \left(-2 \gamma X_{r}^{\pi^{*}}\right)<+\infty
$$

for all $(x, y, t) \in \mathbb{R}^{2} \times[0, T]$.

Since $\pi^{*} \sigma$ is bounded, the Novikov condition holds, i.e.

$$
\mathbb{E}_{y, t} \exp \left(\frac{1}{2} \int_{t}^{T} 4 \gamma^{2}\left(\pi_{s}^{*}\left(Y_{s}, s\right)\right)^{2} \sigma^{2}\left(Y_{s}\right) d s\right)<+\infty .
$$

Hence, the process

$$
\exp \left(-\frac{1}{2} \int_{t}^{r} 4 \gamma^{2} \pi_{s}^{*}\left(Y_{s}, s\right) \sigma^{2}\left(Y_{s}\right) d s-\int_{t}^{r} 2 \gamma \pi_{s}^{*}\left(Y_{s}, s\right) \sigma\left(Y_{s}\right) d W_{s}^{1}\right), \quad t \leq r \leq T,
$$

is a martingale and it follows from the standard martingale Doob inequalities (Revuz and Yor [14, Chapter II, Theorem 1.7]) that

$$
\begin{aligned}
\mathbb{E}_{y, t} \sup _{t \leq r \leq T} \exp \left(-\frac{1}{2} \int_{t}^{r} \gamma^{2}\left(\pi_{s}^{*}\left(Y_{s}, s\right)\right)^{2} \sigma^{2}\left(Y_{s}\right) d s\right. & \\
& \left.-\int_{t}^{r} \gamma \pi_{s}^{*}\left(Y_{s}, s\right) \sigma\left(Y_{s}\right) d W_{s}^{1}\right)<+\infty .
\end{aligned}
$$

Boundedness of $\pi^{*} b$ and $\pi^{*} \sigma$ yields

$$
\begin{aligned}
\mathbb{E}_{x, y, t} & \sup _{t \leq r \leq T} \exp \left(-\gamma X_{r}^{\pi^{*}}\right)=\mathbb{E}_{y, t} \sup _{t \leq r \leq T}(\exp (-2 \gamma x) \\
& \times \exp \left(-\int_{t}^{r} 2 \gamma \pi_{s}^{*}\left(Y_{s}, s\right) b\left(Y_{s}\right) d s+\int_{t}^{r} 2 \gamma^{2}\left(\pi_{s}^{*}\left(Y_{s}, s\right)\right)^{2} \sigma^{2}\left(Y_{s}\right) d s\right) \\
& \left.\times \exp \left(-\int_{t}^{r} 2 \gamma^{2}\left(\pi_{s}^{*}\left(Y_{s}, s\right)\right)^{2} \sigma^{2}\left(Y_{s}\right) d s-\int_{t}^{r} 2 \gamma \pi_{s}^{*}\left(Y_{s}, s\right) \sigma\left(Y_{s}\right) d W_{s}^{1}\right)\right)<+\infty .
\end{aligned}
$$

Applying Proposition 4.1 to $\alpha$ and $\eta^{*}$ shows that

$$
V(y, t)=e^{-\gamma x}(\alpha(y, t))^{1 /\left(1-\rho^{2}\right)}
$$

and $\left(\pi^{*}(y, t), \eta^{*}(y, t)\right)$ satisfy the assumptions of Proposition 3.1. Consequently, the pair $\left(\pi^{*}(y, t), \eta^{*}(y, t)\right)$ is optimal. 
Now our aim is to study our differential game problem under an additional hypothesis. We assume that the set $\Gamma$ is a rectangle $[A, B] \times[C, D]$. It is useful to introduce a function $m$ given by

$$
m(y)= \begin{cases}A & \text { if }-\lambda(y) \leq A \\ -\lambda(y) & \text { if }-\lambda(y) \in[A, B] \\ B & \text { if }-\lambda(y) \geq B\end{cases}
$$

and the set $\overline{\mathcal{M}}$ of all progressively measurable processes $\eta$ with values in $[C, D]$.

Corollary 5.4. If $\Gamma=[A, B] \times[C, D]$ then the maximum in (5.6) is achieved at $\eta_{1}^{*}=m(y), \eta_{2}^{*} \in\{C, D\}$ and

$$
\begin{aligned}
\alpha_{t}+\frac{1}{2} \alpha_{y y}-\frac{1-\rho^{2}}{2}(\lambda(y) & +m(y))^{2} \alpha \\
& +\max _{\eta_{2} \in\{C, D\}}\left(g(y)+\sqrt{1-\rho^{2}} \eta_{2}-\rho \lambda(y)\right) \alpha_{y}=0 .
\end{aligned}
$$

Below, we derive a stochastic representation for the value function $V$. From the classical verification theorem we have

Corollary 5.5. Let $g, \beta, \lambda, \lambda^{2}$ be bounded and uniformly Lipschitz continuous functions. Then

$$
\sup _{\pi \in \mathcal{A}_{t}} \inf _{Q \in \mathcal{Q}} J_{\beta}^{\pi, Q}(x, y, t)=\inf _{Q \in \mathcal{Q}} \sup _{\pi \in \mathcal{A}_{t}} J_{\beta}^{\pi, Q}(x, y, t)=-e^{-\gamma x}(\alpha(y, t))^{1 /\left(1-\rho^{2}\right)},
$$

where

$$
\begin{aligned}
& \alpha(y, t) \\
& \quad=\sup _{\eta \in \mathcal{M}} \mathbb{E}_{y, t} \exp \left(\gamma\left(1-\rho^{2}\right) \beta\left(Y_{T}^{\eta}\right)-\frac{1-\rho^{2}}{2} \int_{t}^{T}\left(\lambda\left(Y_{s}^{\eta}\right)+m\left(Y_{s}^{\eta}\right)\right)^{2} d s\right)
\end{aligned}
$$

and $Y_{t}^{\eta}$ denotes the strong solution of

$$
d Y_{t}=\left(g\left(Y_{t}\right)-\rho \lambda\left(Y_{t}\right)+\sqrt{1-\rho^{2}} \eta_{t}\right) d t+\rho d W_{t}^{1}+\sqrt{1-\rho^{2}} d W_{t}^{2} .
$$

Moreover, the optimal strategy for the agent is given by

$$
\pi^{*}(x, y, t)=\frac{\rho}{\gamma \sigma(y)} \frac{F_{y}}{F}+\frac{\lambda(y)+m(y)}{\gamma \sigma(y)} .
$$

Remark. It follows from the Girsanov theorem that the stochastic representation of $\alpha$ can be rewritten in the following form:

$$
\alpha(y, t)=\sup _{Q \in \overline{\mathcal{Q}}} \mathbb{E}_{y, t}^{Q} \exp \left(\gamma\left(1-\rho^{2}\right) \beta\left(Y_{T}\right)-\frac{1-\rho^{2}}{2} \int_{t}^{T}\left(\lambda\left(Y_{s}\right)+m\left(Y_{s}\right)\right)^{2} d s\right),
$$

where

$$
d Y_{t}=g\left(Y_{t}\right) d t+\rho d W_{t}^{1}+\sqrt{1-\rho^{2}} d W_{t}^{2},
$$


and

$$
\overline{\mathcal{Q}}=\left\{Q \sim P \mid \frac{d Q}{d P}=\mathcal{E}\left(\int-\lambda\left(Y_{t}\right) d W_{t}^{1}+\eta_{t} d W_{t}^{2}\right)_{T}, \eta \in \overline{\mathcal{M}}\right\} .
$$

To conclude, let us investigate the pure investment problem $(\beta \equiv 0)$. Suppose that $(-\lambda(y)) \in[A, B]$ when $y$ belongs to some open set. In that case $\lambda(y)+m(y)=0$ and it follows from the stochastic representation (5.8) that $\pi^{*}(x, y, t)=0$. Therefore if the model uncertainty is large (the interval $[A, B]$ is large), then the best possible strategy is not to invest in the risky asset at all. Moreover, if $(-\lambda(y)) \in[A, B]$ for all $y \in \mathbb{R}$ then the dynamics of $S_{t}$ under the measure $Q^{*}$ is given by

$$
d S_{t}=\sigma\left(Y_{t}\right) d W_{t}^{*}
$$

Hence, a martingale measure is chosen to be an optimal strategy for the market.

\section{References}

[1] F. E. Benth and K. H. Karlsen, A PDE representation of the density of the minimal entropy martingale measure in stochastic volatility markets, Stochastics 77 (2005), 109-137.

[2] W. Fleming and H. M. Soner, Controlled Markov Processes and Viscosity Solutions, 2nd ed., Springer, New York, 2006.

[3] H. Föllmer and A. Gundel, Robust projections in the class of martingale measures, Illinois J. Math. 50 (2006), 439-472.

[4] A. Friedman, The Cauchy problem for first order partial differential equations, Indiana Univ. Math. J. 23 (1973), 27-40.

[5] A. Gundel, Robust utility maximization for complete and incomplete market models, Finance Stoch. 9 (2005), 151-176.

[6] D. Hernández and A. Schied, Robust utility maximization in a stochastic factor models, Statist. Decisions 24 (2006), 109-125.

[7] R. Korn and O. Menkens, Worst-case scenario portfolio optimization: a new stochastic control approach, Math. Methods Oper. Res. 62 (2005), 123-140.

[8] R. Korn and P. Wilmott, Optimal portfolios under the threat of a crash, Int. J. Theor. Appl. Finance 5 (2002), 171-187.

[9] S. Mataramvura and B. Øksendal, Risk minimizing portfolios and HJBI equations for stochastic differential games, Stochastics 80 (2008), 317-337.

[10] M. Musiela and T. Zariphopoulou, An example of indifference prices under exponential preferences, Finance Stoch. 8 (2004), 229-239.

[11] B. Øksendal and A. Sulem, A game theoretic approach to martingale measures in incomplete markets, Obozr. Prikl. Prom. Mat. 15 (2008), 18-24.

[12] -, -, Risk indifference pricing in jump diffusion market, Math. Finance 19 (2009), 619-637.

[13] H. Pham, Smooth solutions to optimal investment models with stochastic volatilities and portfolio constraints, Appl. Math. Optim. 46 (2002), 55-78.

[14] D. Revuz and M. Yor, Continuous Martingales and Brownian Motion, 3rd ed., Springer, Berlin, 1999. 
[15] A. Schied, Robust optimal control for a consumption-investment problem, Math. Methods. Oper. Res. 67 (2008), 1-20.

[16] A. Schied and C. T. Wu, Duality theory for optimal investments under model uncertainty, Statist. Decisions 23 (2005), 199-217.

[17] S. Stoikov and T. Zariphopoulou, Optimal investments in the presence of unhedgeable risks and under CARA preferences, IMA Vol. Ser., in press (2009).

[18] D. Talay and Z. Zheng, Worst case model risk management, Finance Stoch. 6 (2002), $517-537$.

[19] D. H. Wagner, Survey of measurable selection theorems, SIAM J. Control Optim. 15 (1977), 859-903.

[20] T. Zariphopoulou, Optimal investment and consumption models with nonlinear stock dynamics, Math. Methods Oper. Res. 50 (1999), 271-296.

[21] -, A solution approach to valuation with unhedgeable risks, Finance Stoch. 5 (2001), 61-82.

Dariusz Zawisza

Institute of Mathematics

Jagiellonian University

Łojasiewicza 6

30-348 Kraków, Poland

E-mail: dariusz.zawisza@im.uj.edu.pl

Received on 8.10.2009;

revised version on 18.1.2010 